\title{
Downregulation of IL6 Targeted MiR-376b May Contribute to a Positive IL6 Feedback Loop During Early Liver Regeneration in Mice
}

\author{
Shan Lua,b Heng Jiaoc Juan Xud Yongxia Zhenge Yimin Sun ${ }^{\mathrm{f}}$ Huan Chen ${ }^{\mathrm{b}}$ \\ aDepartment of Biochemistry and Molecular Biology, Second Military Medical University, Shanghai, \\ bState Key Laboratory of Proteomics, Beijing Proteome Research Center, Beijing Institute of Radiation \\ Medicine, Beijing, 'Department of General Surgery, Zhongshan Hospital, Shanghai Medical College of \\ Fudan University, Shanghai, dDepartment of geriatrics, Hangzhou, Zhejiang, eMedical College, Jiaxing \\ University, Jiaxing, The State Key Laboratory Breeding Base-Shenzhen Key Laboratory of Chemical \\ Biology, The Graduate School at Shenzhen, Tsinghua University, Shenzhen, China
}

\section{Key Words}

MiR-376b - Liver regeneration - Signal transducer and transcription activators 3 • NF-kappa-B inhibitor zeta

\footnotetext{
Abstract

Background/Aims: MicroRNAs (miRNAs) are a group of endogenous, small, noncoding RNAs implicated in a variety of biological processes, including cell proliferation, apoptosis, differentiation and metabolism. The present study aims to explore the potential role and molecular mechanism of miR-376b during the early phase of liver regeneration. Methods: MiRNA profiling microarrays were used to assess the changes in miRNA expression. For functional analysis, cell proliferation, apoptosis assays, real time quantitative PCR and westernblot analysis were performed. Results: The comprehensive miRNA expression profiling assays on regenerating liver tissues $4 \mathrm{~h}$ after partial hepatectomy $(\mathrm{PH})$ showed that three miRNAs (miR-127, miR-376b and miR-494) located in the DIk1-Gtl2 miRNA cluster were significantly downregulated. In vitro functional studies demonstrated that high-level interleukin 6 (IL6) inhibited the expression of miR-376b, and miR-376b mimics treatment decreased cell proliferation and increased apoptosis. Further target analysis showed that miR-376b reduced the mRNA and protein expression levels of NF-kappa-B inhibitor zeta (NFKBIZ) and signal transducers and transcription activators 3 (STAT3). Additionally, IL6induced miR-376b downregulation would, in turn, increase the expression of IL-6 possibly via a feedback loop involving NFKBIZ or/and STAT3. Conclusion: During the early phase of liver regeneration, miR-376b expression was significantly decreased. Our findings reveal that a regulatory circuitry between miR-376b and IL-6 may exist, which trigger the initiation of liver regeneration.

Copyright (C) 2015 S. Karger AG, Basel

S. Lu and H. Jiao contributed equally to this article.

Huan Chen and Yimin Sun

KARGER 125

State Key Laboratory of Proteomics, Beijing Proteome Research Center, Beijing Institute of Radiation Medicine, \#33 Life Science Park Road, Beijing 102206, (China);

The State Key Laboratory Breeding Base-Shenzhen Key Laboratory of Chemical Biology,

The Graduate School at Shenzhen, Tsinghua University, Shenzhen, (China)

E-Mail chenhuansym@163.com, E-Mail ymsun@capitalbio.com
} 


\section{Introduction}

MicroRNAs (miRNAs) are a class of post-transcriptional regulators that play a critical role in liver development and the fine-tuning of fundamental biological processes in the liver, including liver regeneration, metabolic function and hepatocellular carcinoma (HCC) development [1,2]. Recent studies on liver regeneration have identified a number of miRNAs that show a regulated expression pattern [3-6]. Liver regeneration and hepatocyte proliferation is an orchestrated process involving myriads of signals including cytokines, growth factors, transcription factors and cell cycle regulators [7-10]. When there is a massive loss of hepatocytes, activation of hepatic progenitor cells (HPCs) also occurs [11, 12]. Therefore, miRNAs involved in the above signaling pathways are potential important regulators during hepatocyte proliferation. Array-based assays have been widely used in functional study of some miRNAs during liver regeneration. In situ hybridization (ISH) or qPCR showed that the expression of miR-21 was up-regulated hours post hepatectomy, which inhibited Peli1 expression and potentially regulate NF- $\kappa B$ signaling [13], while miR$34 c^{*}$, miR-144, and miR-451 were markedly downregulated in the liver tissues after partial hepatectomy $(\mathrm{PH})[14]$. However, identification of the functional miRNAs in the regenerating liver remains incomplete, partly due to the obscure mechanism underlying liver regeneration in different stages and the large number of possible miRNA:mRNA targeting relationships.

In the present study, we used an array-based assay to screen the deregulated miRNA in regenerating liver tissues (4h) correspond to the G1 phase, which identified that miR-376b, miR-494 and miR-127 was significantly down-regulated $4 \mathrm{~h}$ post-surgery, as compared to the control. Interestingly, the three miRNAs are located in the imprinted Dlk1-Gtl2 imprinted region $12 \mathrm{qF} 1$ [15], map to the human Dlk1-Dio3 region on chromosome 14q32.2, which exert inhibitory effect in HCC malignancy in vitro [16]. The Dlk1-Dio3 region is also a cancer susceptibility locus and dysregulation of the miRNAs in this region has been found in liver tumors [17-19]. The above researches, to date, did not focus on the potential role of the Dlk1Dio3 miRNA clusters during liver regeneration.

Interestingly, enforced interleukin 6 (IL6) production in cholangiocarcinoma cells is able to induce Dlk1-Dio3 domain hypermethylation, which in turn inhibits the expression of Dlk1-Dio3 region-embedded miRNA [20, 21].Since IL6 has been shown to play a major role in the acute phase response after liver injury, we hypothesized that the miR-376b is involved in the IL6 signaling transduction system and hence contribute to the priming phase of liver regeneration.

\section{Materials and Methods}

\section{Ethics Statement}

All C57BL/6 mice were appropriately cared and all animal experiments were performed according to the Institutional Animal Care Instructions approved by the Ethics Committee of the Second Military Medical University (Shanghai, China; Approval ID: SCXK 2007-0003). All animals were fed on a standard laboratory chow with free access to water.

\section{Animals and Surgery}

Male C57BL/6 mice aged 8-10 week were randomly assigned to partial hepatectomy (PH) and a sham operation ( $\mathrm{SH}$ ) group (3 6 animals in each group). After an overnight fast, the mice were injected intraperitoneally (i.p.) with sodium pentobarbital $(30 \mathrm{mg} / \mathrm{kg}$ ) and underwent $70 \%$ PH. Four hours after surgery, the animals were sacrificed to collect the remaining liver tissue for subsequent examination.

\section{Microarrays}

Total RNA samples from SH and PH mice were analyzed by CapitalBio Corporation (CapitalBio, Beijing, China) for miRNA microarray experiments as described before [4]. MiRNAs were separated from 20-30 ug total RNA using the Ambion miRNA Isolation Kit (including small RNAs). Fluorescein-labeled miRNAs 
were used for hybridization on each miRNA microarray chip containing 1320 probes in triplicate. The differentially expressed miRNAs were chosen by using the Significance Analysis of Microarrays program (version 2.1). The alterations were defined as those with either $<0.5$ or $>2$-fold changes with a p value of less than 0.05 . For mRNA expression profiling and data analysis, the mouse genome $35 \mathrm{~K}$ oligonucleotide microarray was constructed and applied at CapitalBio Corporation (Beijing, China), as previously reported.

\section{Cell culture and transfection}

Murine hepatoma Hepa1-6 cells (Institute of Biochemistry and Cell Biology, Shanghai, China) were cultured in DMEM (Biowest, Loire, France) with $10 \%$ fetal bovine serum (FBS, Biowest, Loire, France) in a humidified atmosphere containing $5 \% \mathrm{CO}_{2}$ at $37^{\circ} \mathrm{C}$. MiR-376b mimics and negative control RNA were obtained from GenePharma (Shanghai, China). Transfection was performed using Lipofectamine 2000 (Invitrogen, CA, USA) according to the manufacturer's protocols. Cells were cultured in 6-well plates and treated with IL-6 (10nM) for $4 \mathrm{~h}$ or $8 \mathrm{~h}$. The cells were then collected to analyze the expression of NFKBIZ and STAT3.

\section{Cell proliferation assay}

Hepa1-6 cells were transfected with the miR-376b mimics or the negative control in the 24-well plate. After 24-h transfection, cells were reseeded in 96 -well plates at a density of 4000 cells per well. At various time points (day 2 and 4), $20 \mu \mathrm{l}$ methylthiazoletetrazolium (MTT) solution $(5 \mathrm{mg} / \mathrm{mL}$ ) was added into the culture medium for 4-h incubation. Then $150 \mu \mathrm{l}$ dimethyl sulfoxide (DMSO) was added to each well to dissolve the crystals. After 10-min dissolution, the absorbance of each sample was recorded at $570 \mathrm{~nm}$. The experiment was performed in triplicate.

Apoptosis assay

Hepa1-6 cells were plated in 6-well plates and transfected with the miR-376b mimics or the negative control. After 48-h incubation, the cells were collected for apoptosis analysis by flow cytometry. The number of apoptotic cells was determined with the annexin V/ FITC detection kit (Keygentec, Nanjing, China) according to the manufacturer's instructions. Data acquisition and analysis were performed using a FACSort Cytometer (FACSCA, New York, USA). The apoptotic cell rate was the sum of the early and the late apoptotic cells.

\section{QRT-PCR}

Total RNA was isolated from the prepared liver samples and cells using TRIzol reagent (Invitrogen, Carlsbad, CA, USA). cDNA was synthesized following the manufacturer' protocols (MBI Fermentas, Vilnius, Lithuania). QRT-PCR was performed with a standard SYBR-green PCR kit (TOYOBO, Osaka, Japan), and genespecific PCR amplification was performed using the ABI 7300 (Applied Biosystems, Darmstadt, Germany). qRT-PCR reactions were performed in triplicate. Relative gene expression was calculated with the $2^{-\Delta \Delta C t}$ method after normalization to the expression of $\beta$-actin or U6. The primers are listed as follows. U6 forward 5'- CTC GCT TCG GCA GCACA-3', U6 reverse 5'- AAC GCT TCA CGA ATT TGCGT-3'; $\beta$-actin forward 5'- TCG CAC AAT GAC TCT GGAAG-3', $\beta$-actin reverse 5'- CAG GGT CCA CAT TCA GGACT-3'; Stat3 forward 5'- GTT TAC CCA GTA TGC TTG TCG-3', Stat3 reverse 5'- CCC AGT AAG GCA CCC ACA-3'; Nfkbiz forward 5'- CCA GTT GCC TGT CTT TCG-3', Nfkbiz reverse 5'- CGG GCT GTT CAT TCT CCA-3'; IL6 forward 5'- TTG CCT TCT TGG GAC TGA-3', IL6 reverse 5'- TTG CCA TTG CAC AAC TCTT-3'.

\section{Western blotting analysis}

Cells treated with miR-376b were lysed in RIPA lysis buffer (Beyotime, Jiangsu, China). The lysates were centrifuged at $12,000 \mathrm{rpm}$ at $4{ }^{\circ} \mathrm{C}$ for $10 \mathrm{~min}$. Equal amounts of protein were separated using $10-15 \%$ sodium dodecyl sulfonate-polyacrylamide gel electrophoresis and then transferred to nitrocellulose membranes (Boguang, Shanghai, China) subsequently. For immunoblotting, the membranes were respectively incubated with antibodies specific for NFKBIZ (Epitomics, Burlingame, CA), STAT3 (Abcam, Cambridge, UK), p-STAT3 (Epitomics, Burlingame, CA) and $\beta$-actin (Epitomics, Burlingame, CA). The immunoblotting sample was incubated with horseradish peroxidase (HRP)-coupled anti-rabbit secondary antibodies (ProteinTech, Chicago, USA) and visualized using enhanced chemiluminescence (Pierce, Rockford, USA). 
Statistics

Statistical analysis was performed by using the SPSS statistics software package (SPSS). All results were expressed as mean $\pm \mathrm{SD}$, and $\mathrm{P}<0.05$ was used for significance.

\section{Results}

MiR-376b is downregulated in response to $\mathrm{PH}$

To illustrate the miRNA expression pattern in the early phase of liver regeneration in mice, a comprehensive miRNA profiling microarray analysis was performed on regenerating liver tissues at $4 \mathrm{~h}$ post-surgery. It was found that four miRNAs were upregulated and three miRNAs were downregulated (Fig. 1A). Interestingly, all three downregulated miRNAs (miR-127, miR-376b and miR-494) were located in the mouse $12 \mathrm{qF} 1$ region, also known as Dlk1- Gtl2 region. This Dlk1- Gtl2 imprinted locus contains three protein-coding genes from the paternally inherited chromosome [delta-like homologue 1 (DLK1), retrotransposonlike gene 1 (RTL1), and the type 3 deiodinase (DIO3)], and several noncoding RNA genes including miRNAs, snoRNAs and a large noncoding RNA Gtl2 (Fig. 1B) [15]. Our QRT-PCR analysis further confirmed that the expression levels of miR-127, miR-376b and miR-494 were significantly decreased $(<0.5$ fold $)$ in regenerating liver tissues.

Effect of IL6 regulated miR-376b on hepatocyte proliferation and apoptosis

It is known that IL6 can induce the hypermethylation of Dlk1-Dio3 domain and downregulate the expression of clustered miRNA [20,21]. We speculated that IL6 may play a role in regulating miR-376b expression in hepatocytes. Our mRNA microarray analysis showed that 17 genes in the IL6/Jak/STAT signaling pathway were differentially expressed 4 $\mathrm{h}$ after PH (Table 1), suggesting that overexpressed IL6 may contribute to the downregulation of miR-376b expression during liver regeneration. Moreover, we confirmed that ectopic IL6

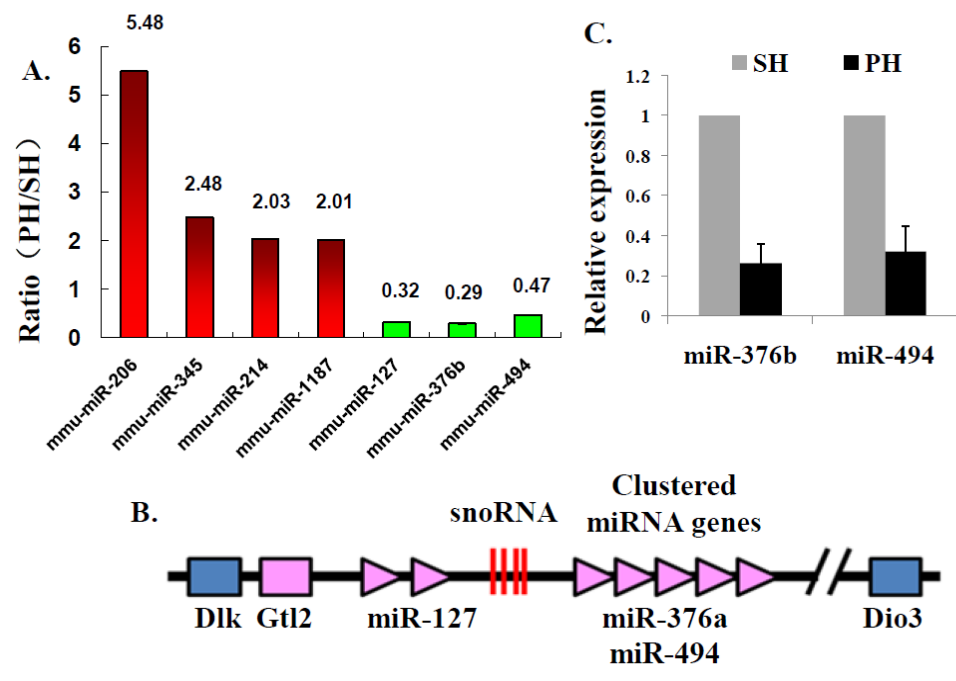

Fig. 1. Differentially expressed miRNAs during liver regeneration (LR). (A) miRNA expression profiling at $4 \mathrm{~h}$ after partial hepatectomy (PH). Each data point represents the ratio of miRNA expression levels under PH to Sham operation (SH). Ratio values $>1.5$ or $<0.67$ were considered up-regulated (red) or downregulated (green) in $\mathrm{PH}$ rats compared to $\mathrm{SH}$ rats. (B) The location of miR-127, miR-376b and miR-494 in Dlk1- Gtl2 region. (C) The expression pattern of miR-127, miR-376b and miR-494 during liver regeneration by quantitative real-time PCR (qRT-PCR) analysis. The data were obtained from at least three independent experiments and analyzed by t test. 
significantly inhibited the expression of miR-376b in the murine hepatoma Hepa1-6 cell model, as well as two other miRNAs located in the Dlk-Dio3 domain (Fig. 2A).

To evaluate the biological effect of miR-376b in regulating murine liver cell proliferation, a MTT cell proliferation assay was employed in Hepa1-6 cells. After treatment with miR-376b mimics, cell growth was strongly repressed at day 4 , as compared to the control group. In addition, the apoptotic effect was increased in the miR-376b treated cells (Fig. 1C). These data suggest thatmiR-376 working as a growth inhibitor may play an inhibitory role in the IL6 promitogenic axis in hepatocyte proliferation.

MiR-376b suppresses NFKBIZ and STAT3 expression

In order to identify the target genes and underlying molecular mechanism by which miR-376b regulates liver cell growth, we analyzed the intersection between differentially expressed mRNAs during early liver regeneration and bioinformatic target analysis (TargetScan and RNA22) on miR-376b. We identified two potential target genes, NF-kappa-B inhibitor zeta (NFKBIZ) and STAT3 (Fig. 3A), both of which have been reported to associate with the activation of IL6 signaling $[22,23]$. We then carried QRT-PCR and Westernblot analysis to see whether miR-376b could regulate the expression of both genes. As shown in Fig. 3B-D, miR$376 \mathrm{~b}$ mimics drastically inhibited the expression of NFKBIZ and STAT3 on both mRNA and protein levels. Conversely, miR-376b inhibitor significantly increased the protein levels of both genes. These data strongly
Table 1. Deregulated mRNA (in the IL6/ Jak/STAT signaling pathway) at $4 \mathrm{~h}$ post $\mathrm{PH}$

\begin{tabular}{ccc}
\hline RefSeq & Name & Ratio \\
\hline NM_009896 & Socs1 & 7.1165 \\
NM_009971 & Csf3 & 6.9822 \\
NM_009895 & Cish & 6.4476 \\
NM_007707 & Socs3 & 5.5829 \\
NM_019667 & Stam2 & 4.8267 \\
NM_008842 & Pim1 & 4.6271 \\
NM_031168 & Il6 & 4.0221 \\
NM_010849 & Myc & 3.8774 \\
NM_010557 & Il4ra & 3.0129 \\
NM_007706 & Socs2 & 2.8679 \\
NM_008348 & Il10ra & 2.3978 \\
NM_009743 & Bcl211 & 2.3108 \\
NM_007781 & Csf2rb2 & 2.2999 \\
NM_011486 & Stat3 & 2.1033 \\
NM_008358 & Il15ra & 0.4935 \\
NM_011085 & Pik3r1 & 0.4007 \\
NM_008353 & Il12rb1 & 0.2643 \\
\hline & & \\
\hline
\end{tabular}

Fig. 2. IL6 regulated miR376b inhibited cell proliferation and induced cell apoptosis in Hepa1-6 cell. (A) IL-6 treatment for $4 \mathrm{~h}$ decreased the expression of miR-376b, miR-127 and miR-494 in Hepa1-6 cell by qRT-PCR. (B) Hepa1-6 cell transfected with miR-376b or negative control (NC) were seeded in 96-well plates and examined at indicated time points. The absorbance of methylthiazoletetrazolium was recorded at 570 $\mathrm{nm}$ after staining. (C and D) Cells treated with miR-376b or NC were analyzed by flow
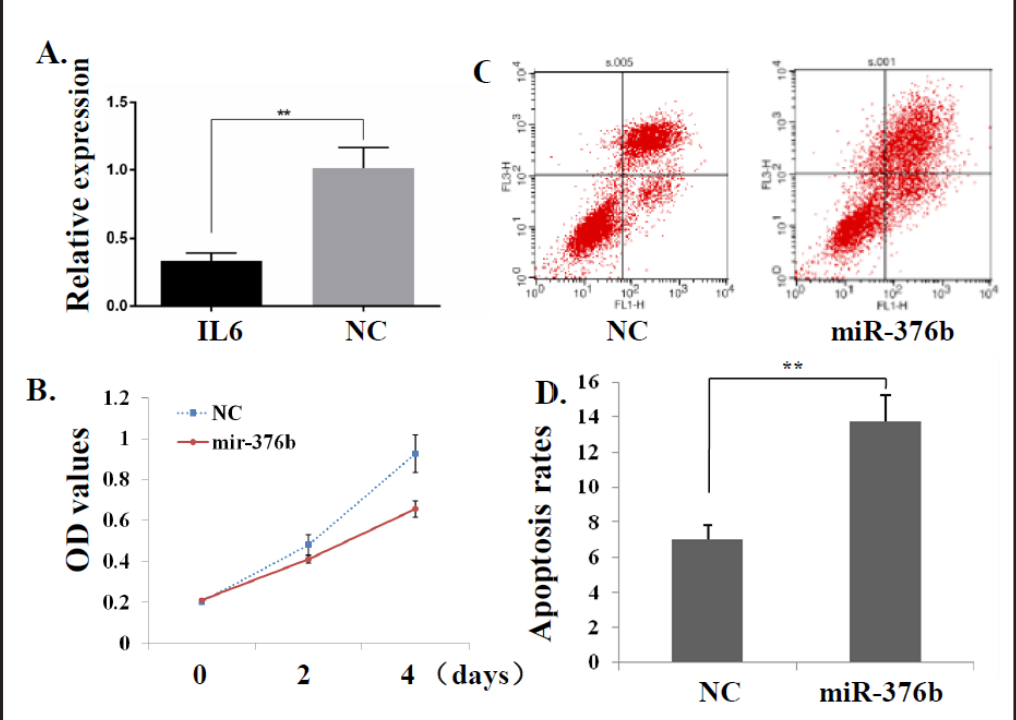
cytometry. The data were obtained from at least three independent experiments and analyzed by $t$ test $\left(* \mathrm{P}<0.05,{ }^{*} \mathrm{P}<0.01\right)$. 


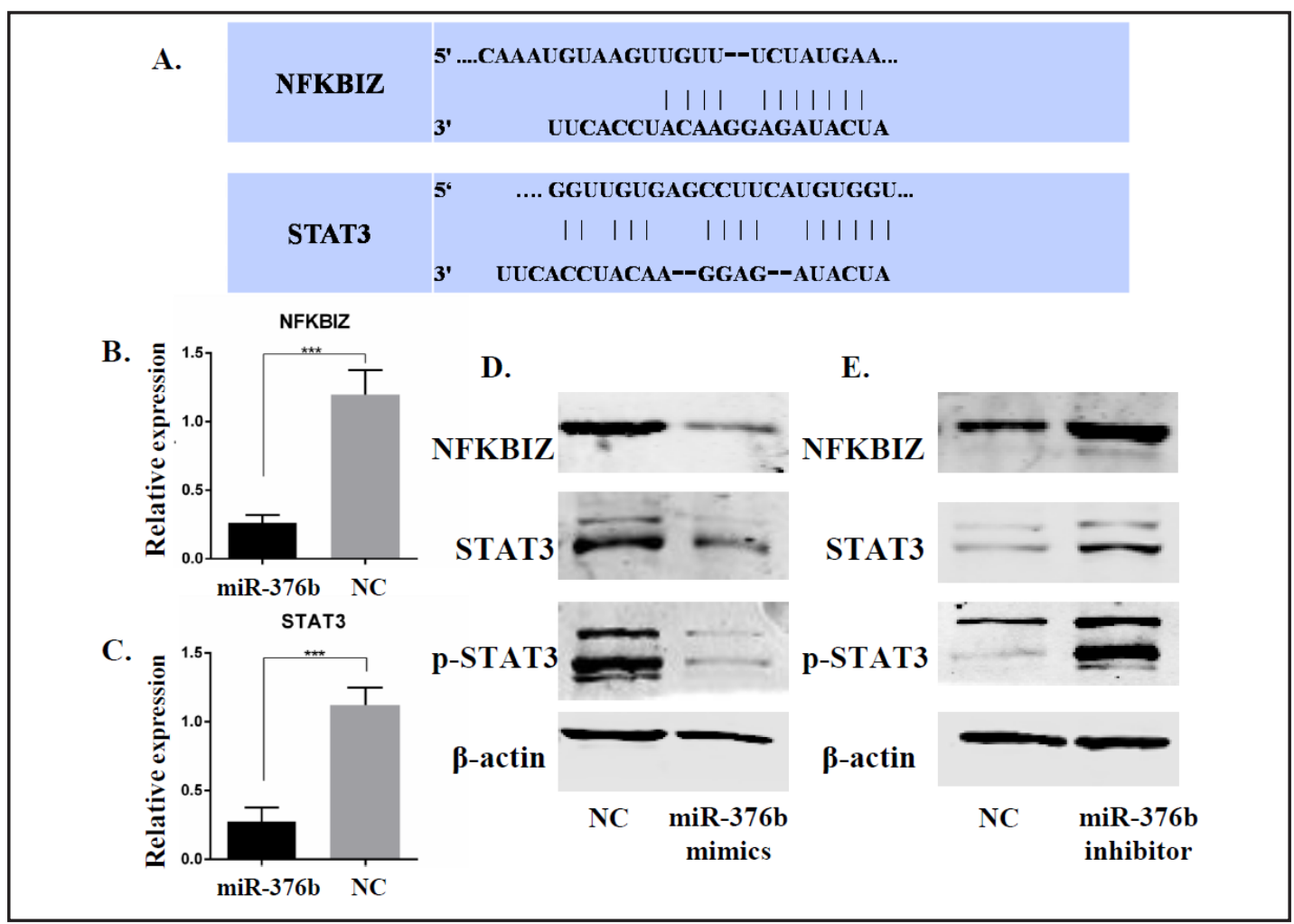

Fig. 3. Analyses of candidate target genes of miR-376b. (A) miR-376b-binding sites in NFKBIZ and STAT3 predicted by TargetScan and RNA22, respectively. (B) miR-376b decreased mRNA expression of NF-kappa-B inhibitor zeta (NFKBIZ) by qRT- PCR. (C) miR-376b decreased mRNA expression of signal transducers and transcription activators 3 (STAT3) by qRT- PCR. (D) miR-376b mimics decreased protein expression of NFKBIZ and STAT3 by western blot analysis. $\beta$-actin was used as sample control. (E) miR-376b inhibitor increased protein expression of NFKBIZ and STAT3 by western blot analysis. $\beta$-actin was used as sample control. NT stands for no treatment. The data were obtained from at least three independent experiments and analyzed by t test $(* * * \mathrm{P}<0.001)$.

suggest that miR-376b may target NFKBIZ and STAT3 in hepatocytes, and the downregulation of miR-376b in the regenerating liver tissue may lead to the increased expression of NFKBIZ and STAT3.

NFKBIZ and STAT3 may contribute to the miR-376b/IL6 positive feedback loop

STAT3 is a known target of IL6 signaling [22]. It was found in our study that ectopic IL6 treatment strongly increase the mRNA expression levels of STAT3, and miR-376b mimics largely blocked the effect of IL6 (Fig. 4A). The same effect of IL6 alone and its combination treatment with miR-376b mimics on NFKBIZ expression was observed in Hepa1-6 cell models (Fig. 4B), indicating that IL6 may upregulate both downstream genes through miR$376 \mathrm{~b}$ dependent posttranscriptional mechanism.

Knowing that p-STAT3 is a positive regulator of IL6 expression [22] and the protein levels of p-STAT3 increases with miR-376b decreasing, we hypothesized that the suppression on miR-376b by IL6 overexpression would consequently upregulate p-STAT3 and IL6 expression. The hypothesis was confirmed by the data in Fig. 4C-E, showing that miR376b mimics inhibited the mRNA expression and supernatant levels of IL6 reciprocally. Our data also showed that overexpression of NFKBIZ could also abrogate the inhibitory effect of miR376b on IL6 production, suggesting a miR-376b/NFKBIZ/IL6 feedback loop. Therefore, the present study revealed that both miR-376b downstream genes (NFKBIZ and STAT3) contributed to the IL6/miR-376B/IL6 feedback loop in hepatocytes.

\section{KARGER}



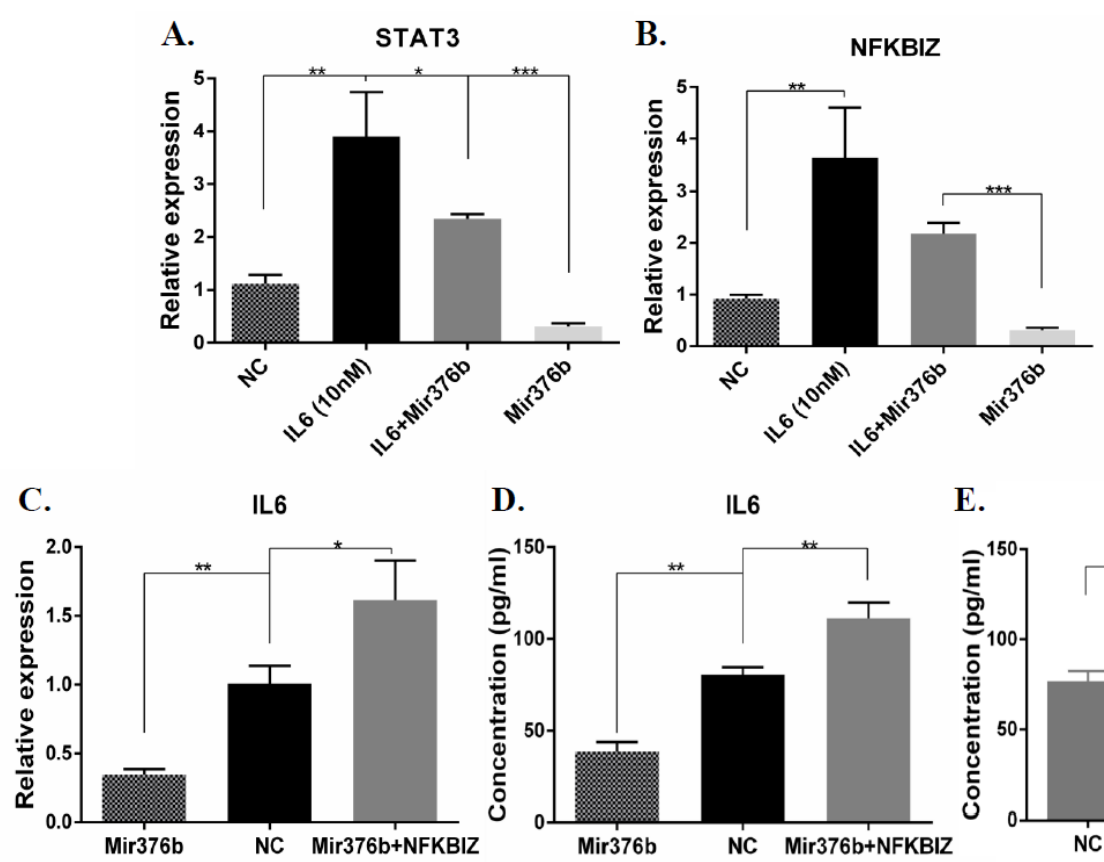

D.

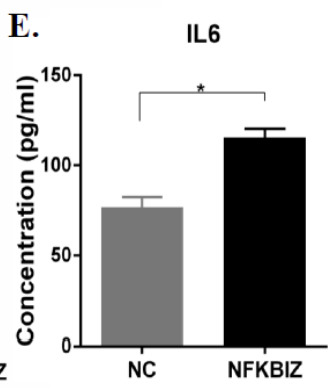

Fig. 4. Correlation between the expression levels of IL6, miR-376b, STAT3 and NFKBIZ. (A) IL6 treatment increased the mRNA expression levels of STAT3, and miR-376b mimics blocked the effect of IL6. (B) IL6 treatment increased the mRNA expression levels of NFKBIZ, and miR-376b mimics blocked the effect of IL6. (C, D) miR376b mimics inhibited the mRNA expression and IL6 levels in culture supernatant, overexpression of NFKBIZ abrogated the inhibitory effect of miR-376b on IL6 production. (E) NFKBIZ alone increases the expression of IL6. The data were obtained from at least three independent experiments and analyzed by $t$ test $\left(* \mathrm{P}<0.05, * * \mathrm{P}<0.01,{ }^{* * *} \mathrm{P}<0.001\right)$.

\section{Discussion}

The mRNA array results in the present study showed that the Jak-STAT3 pathway was one of the essential signaling pathway during early liver regeneration (Table 1), which agrees with the previous finding that IL6 secreted by kupffer cells stimulated the initiation of liver regeneration [24, 25]. At the same time, microRNA array revealed that three Dlk-Dio3 region-imprinted miRNAs (miR-494, miR-376a and miR-127) were downregulated. IL6 has been shown to be a negative regulator of Dlk1-Dio3 cluster [20]. We therefore hypothesized that the
Fig. 5. The feedforward loop comprised of IL-6, miR376b, STAT3 and NFKBIZ.

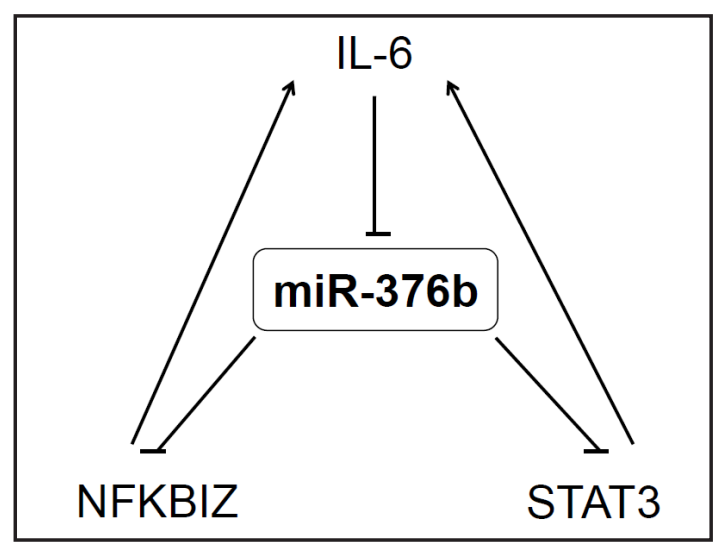
downregulation of miR-376 and two other miRNAs may be attributed to the activation of IL6 during early liver regeneration. Of note, we confirmed that IL6 treatment could downregulate the expression of all three miRNAs on the cellular model (Fig. 2A). In human, miR-376, miR127 and miR-494 have been reported to be downregulated in several cancers, including human hepatocellular carcinoma (HCC), breast cancer and oral cancers. Overexpression of these miRNAs in cancer cells may lead to suppressed cell growth or induced apoptosis [26-29]. As a promitogenic signal shortly activated after PH, IL6 plays an essential role in triggering the "initiation signal" for hepatocytes to reenter G1 phase and cell cycle progression. 
Next, we confirmed that miR-376b had an anti-proliferative effect by increasing apoptosis and decreasing liver cell growth (Fig. 2). Thus, we assumed that when IL6 is induced post $\mathrm{PH}$, the downstream pro-proliferative Jak/STAT pathway is activated and the anti-proliferative miR-376b signaling pathway is inhibited, which cooperatively contribute to liver regeneration progression.

Moreover, we identified that NFKBIZ and STAT3, the already known pro-inflammatory gene and oncogene respectively [30,31], were the downstream target of miR-376b (Fig. 3 ). Thus, it is deemed that down-regulation of miR-376b during early phase triggers cell proliferation by upregulating the mRNA and protein levels of NFKBIZ and STAT3. Interestingly, we also observed a significant decrease in pSTAT3 expression by miR-376b overexpression. Since both the mRNA and protein expression levels of STAT3 were strongly repressed by miR-376b mimics treatment, we presumed that pSTAT3 level was changed accordingly when total STAT3 expression was decreased. Furthermore, accumulating evidence suggests that NFKBIZ and STAT3 can induce the expression of IL-6 as transcription factor $[18,23]$. Then IL-6, miR-376b, STAT3 and NFKBIZ comprised a feedforward loop (Fig. 5), which links the output of a circuit back to its input. Namely, IL-6 secreted by kupffer cells may decrease the expression of miR-376b in hepatocyte cells, resulting in activation of NFKBIZ and STAT3, and promote the proliferation of hepatocytes.

Feed-forward regulation expands the set of possible biological properties and allows the system to convert graded inputs into decisive, all-or-none outputs [32]; while the feature of positive feedback loops cover signal amplification, bistability and memory module [3335]. Hence, the positive feedback loop mediated by miR-376b may significantly amplify the IL- 6 signaling and reinforce the proliferation caused by IL- 6 activation. Our hypothesis could explain, as least in part, why the liver responds to injury so rapidly and robustly. Altogether, IL-6/miR-376b/STAT3 and IL-6/miR-376b/NFKBIZ feedback loop may cooperatively contribute to the initiation stage of liver regeneration. In summary, our findings reveal a regulatory circuitry between miR-376b and IL-6 during the early phase of liver regeneration.

\section{Abbreviations}

PH (partial hepatectomy); IL6 (interleukin 6); NFKBIZ (NF-kappa-B inhibitor zeta); STAT3 (signal transducers and transcription activators 3); SH (sham operation); miRNAs (microRNAs).

\section{Acknowledgments}

This work was financially supported by the National Science Foundation of China [No. 31371362, No. 81171920 and No. 81400654].

\section{Disclosure Statement}

None.

\section{References}

1 Chen Y, Verfaillie CM: MicroRNAs: the fine modulators of liver development and function. Liver Int 2014;34:976-990.

2 Bandiera S, Pfeffer S, Baumert TF, Zeisel MB: miR-122 - A key factor and therapeutic target in liver disease. J Hepatol 2015;62:448-457. 
3 Werner W, Sallmon H, Leder A, Lippert S, Reutzel-Selke A, Morgul MH, Jonas S, Dame C, Neuhaus P, Iacomini J, Tullius SG, Sauer IM, Raschzok N: Independent effects of sham laparotomy and anesthesia on hepatic microRNA expression in rats. BMC Res Notes 2014;7:702.

4 Chen H, Sun Y, Dong R, Yang S, Pan C, Xiang D, Miao M, Jiao B: Mir-34a is upregulated during liver regeneration in rats and is associated with the suppression of hepatocyte proliferation. PLoS One 2011;6:e20238.

5 Shu J, Kren BT, Xia Z, Wong PY, Li L, Hanse EA, Min MX, Li B, Albrecht JH, Zeng Y, Subramanian S, Steer CJ: Genomewide microRNA down-regulation as a negative feedback mechanism in the early phases of liver regeneration. Hepatology 2011;54:609-619.

6 Song G, Sharma AD, Roll GR, Ng R, Lee AY, Blelloch RH, Frandsen NM, Willenbring H: MicroRNAs control hepatocyte proliferation during liver regeneration. Hepatology;51:1735-1743.

7 Zimmermann A: Regulation of liver regeneration. Nephrol Dial Transplant 2004;19:iv6-10.

8 Akita K, Okuno M, Enya M, Imai S, Moriwaki H, Kawada N, Suzuki Y, Kojima S: Impaired liver regeneration in mice by lipopolysaccharide via TNF-alpha/kallikrein-mediated activation of latent TGF-beta. Gastroenterology 2002;123:352-364.

9 Taub R: Liver regeneration: from myth to mechanism. Nat Rev Mol Cell Biol 2004;5:836-847.

10 Liu S, Wierod L, Skarpen E, Grosvik H, Duan G, Huitfeldt HS: EGF activates autocrine TGFalpha to induce prolonged egf receptor signaling and hepatocyte proliferation. Cell Physiol Biochem 2013;32:511-522.

11 Katoonizadeh A, Poustchi H, Malekzadeh R: Hepatic progenitor cells in liver regeneration: current advances and clinical perspectives. Liver Int 2014;34:1464-1472.

12 Bi Y, He Y, Huang J, Su Y, Zhu GH, Wang Y, Qiao M, Zhang BQ, Zhang H, Wang Z, Liu W, Cui J, Kang Q, Zhang Z, Deng Y, Li R, Zhang Q, Yang K, Luu HH, Haydon RC, He TC, Tang N: Functional characteristics of reversibly immortalized hepatic progenitor cells derived from mouse embryonic liver. Cell Physiol Biochem 2014;34:1318-1338.

13 Marquez RT, Wendlandt E, Galle CS, Keck K, McCaffrey AP: MicroRNA-21 is upregulated during the proliferative phase of liver regeneration, targets Pellino-1, and inhibits NF-kappaB signaling. Am J Physiol Gastrointest Liver Physiol 2010;298:G535-541.

14 Chaveles I, Zaravinos A, Habeos IG, Karavias DD, Maroulis I, Spandidos DA, Karavias D: MicroRNA profiling in murine liver after partial hepatectomy. Int J Mol Med 2012;29:747-755.

15 Seitz H, Royo H, Bortolin ML, Lin SP, Ferguson-Smith AC, Cavaille J: A large imprinted microRNA gene cluster at the mouse Dlk1-Gtl2 domain. Genome Res 2004;14:1741-1748.

16 Benetatos L, Hatzimichael E, Londin E, Vartholomatos G, Loher P, Rigoutsos I, Briasoulis E: The microRNAs within the DLK1-DIO3 genomic region: involvement in disease pathogenesis. Cell Mol Life Sci 2013;70:795-814.

17 Cheunsuchon P, Zhou Y, Zhang X, Lee H, Chen W, Nakayama Y, Rice KA, Tessa Hedley-Whyte E, Swearingen B, Klibanski A: Silencing of the imprinted DLK1-MEG3 locus in human clinically nonfunctioning pituitary adenomas. Am J Pathol 2011;179:2120-2130.

18 Lempiainen H, Couttet P, Bolognani F, Muller A, Dubost V, Luisier R, Del Rio Espinola A, Vitry V, Unterberger EB, Thomson JP, Treindl F, Metzger U, Wrzodek C, Hahne F, Zollinger T, Brasa S, Kalteis M, Marcellin M, Giudicelli F, Braeuning A, Morawiec L, Zamurovic N, Langle U, Scheer N, Schubeler D, Goodman J, Chibout SD, Marlowe J, Theil D, Heard DJ, Grenet O, Zell A, Templin MF, Meehan RR, Wolf RC, Elcombe CR, Schwarz M, Moulin P, Terranova R, Moggs JG: Identification of Dlk1-Dio3 imprinted gene cluster noncoding RNAs as novel candidate biomarkers for liver tumor promotion. Toxicol Sci 2013;131:375-386.

19 Luk JM, Burchard J, Zhang C, Liu AM, Wong KF, Shek FH, Lee NP, Fan ST, Poon RT, Ivanovska I, Philippar U, Cleary MA, Buser CA, Shaw PM, Lee CN, Tenen DG, Dai H, Mao M: DLK1-DIO3 genomic imprinted microRNA cluster at 14q32.2 defines a stemlike subtype of hepatocellular carcinoma associated with poor survival. J Biol Chem 2011;286:30706-30713.

20 Meng F, Wehbe-Janek H, Henson R, Smith H, Patel T: Epigenetic regulation of microRNA-370 by interleukin-6 in malignant human cholangiocytes. Oncogene 2008;27:378-386.

21 An F, Yamanaka S, Allen S, Roberts LR, Gores GJ, Pawlik TM, Xie Q Ishida M, Mezey E, Ferguson-Smith AC, Mori Y, Selaru FM: Silencing of miR-370 in human cholangiocarcinoma by allelic loss and interleukin-6 induced maternal to paternal epigenotype switch. PLoS One 2012;7:e45606. 


\section{Cellular Physiology Cell Physiol Biochem 2015;37:233-242

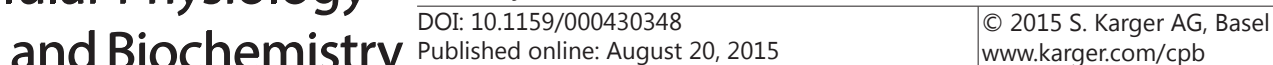

Lu et al.: Mir-376b Involved in IL6 Feedback Loop During Liver Regeneration

22 Chang Q, Bournazou E, Sansone P, Berishaj M, Gao SP, Daly L, Wels J, Theilen T, Granitto S, Zhang X, Cotari J, Alpaugh ML, de Stanchina E, Manova K, Li M, Bonafe M, Ceccarelli C, Taffurelli M, Santini D, Altan-Bonnet G, Kaplan R, Norton L, Nishimoto N, Huszar D, Lyden D, Bromberg J: The IL-6/JAK/Stat3 feed-forward loop drives tumorigenesis and metastasis. Neoplasia 2013;15:848-862.

23 Kitamura H, Kanehira K, Okita K, Morimatsu M, Saito M: MAIL, a novel nuclear I kappa B protein that potentiates LPS-induced IL-6 production. FEBS Lett 2000;485:53-56.

24 Fausto N, Campbell JS, Riehle KJ: Liver regeneration. Hepatology 2006;43:S45-53.

25 Kawai M, Naruse K, Komatsu S, Kobayashi S, Nagino M, Nimura Y, Sokabe M: Mechanical stress-dependent secretion of interleukin 6 by endothelial cells after portal vein embolization: clinical and experimental studies. J Hepatol 2002;37:240-246.

26 Liborio-Kimura TN, Jung HM, Chan EK: miR-494 represses HOXA10 expression and inhibits cell proliferation in oral cancer. Oral Oncol 2015;51:151-157.

27 Wang S, Li H, Wang J, Wang D, Yao A, Li Q: Prognostic and biological significance of microRNA-127 expression in human breast cancer. Dis Markers 2014;2014:401986.

28 Zhou J, Lu S, Yang S, Chen H, Shi H, Miao M, Jiao B: MicroRNA-127 post-transcriptionally downregulates Sept7 and suppresses cell growth in hepatocellular carcinoma cells. Cell Physiol Biochem 2014;33:15371546.

29 Zheng Y, Yin L, Chen H, Yang S, Pan C, Lu S, Miao M, Jiao B: miR-376a suppresses proliferation and induces apoptosis in hepatocellular carcinoma. FEBS Lett 2012;586:2396-2403.

30 Silva CM: Role of STATs as downstream signal transducers in Src family kinase-mediated tumorigenesis. Oncogene 2004;23:8017-8023.

31 Yamazaki S, Muta T, Takeshige K: A novel IkappaB protein, IkappaB-zeta, induced by proinflammatory stimuli, negatively regulates nuclear factor-kappaB in the nuclei. J Biol Chem 2001;276:27657-27662.

32 Brandman 0, Ferrell JE, Jr., Li R, Meyer T: Interlinked fast and slow positive feedback loops drive reliable cell decisions. Science 2005;310:496-498.

33 Venturelli OS, El-Samad H, Murray RM: Synergistic dual positive feedback loops established by molecular sequestration generate robust bimodal response. Proc Natl Acad Sci U S A 2012;109:E3324-3333.

34 Brandman O, Meyer T: Feedback loops shape cellular signals in space and time. Science 2008;322:390-395.

35 Xiong W, Ferrell JE, Jr: A positive-feedback-based bistable 'memory module' that governs a cell fate decision. Nature 2003;426:460-465. 